\title{
Uncertainty quantification and global sensitivity analysis of complex chemical process using a generalized polynomial chaos approach
}

\author{
Pham Luu Trung Duonga ${ }^{a}$, Wahid Ali ${ }^{\mathrm{b}}$, Ezra Kwok ${ }^{\mathrm{c}}$, Moonyong Lee ${ }^{\mathrm{b}, *}$ \\ a Luxembourg Centre for Systems Biomedicine, Systems Control Group, University of Luxembourg, Luxembourg \\ b School of Chemical Engineering, Yeungnam University, Gyeongsan, Republic of Korea \\ ${ }^{\text {c } C h e m i c a l ~ \& ~ B i o l o g i c a l ~ E n g i n e e r i n g, ~ U n i v e r s i t y ~ o f ~ B r i t i s h ~ C o l u m b i a, ~ V a n c o u v e r, ~ C a n a d a ~}$
}

\section{A R T I C L E I N F O}

\section{Article history:}

Received 6 December 2015

Received in revised form 18 February 2016

Accepted 22 March 2016

Available online 29 March 2016

\section{Keywords:}

Generalized polynomial chaos

Uncertainty quantification

Process uncertainty

Sensitivity analysis

Monte-Carlo approach

\begin{abstract}
A B S T R A C T
Uncertainties are ubiquitous and unavoidable in process design and modeling. Because they can significantly affect the safety, reliability and economic decisions, it is important to quantify these uncertainties and reflect their propagation effect to process design. This paper proposes the application of generalized polynomial chaos (gPC)-based approach for uncertainty quantification and sensitivity analysis of complex chemical processes. The gPC approach approximates the dependence of a process state or output on the process inputs and parameters through expansion on an orthogonal polynomial basis. All statistical information of the interested quantity (output) can be obtained from the surrogate gPC model. The proposed methodology was compared with the traditional Monte-Carlo and Quasi Monte-Carlo sampling-based approaches to illustrate its advantages in terms of the computational efficiency. The result showed that the gPC method reduces computational effort for uncertainty quantification of complex chemical processes with an acceptable accuracy. Furthermore, Sobol's sensitivity indices to identify influential random inputs can be obtained directly from the surrogated gPC model, which in turn further reduces the required simulations remarkably. The framework developed in this study can be usefully applied to the robust design of complex processes under uncertainties.
\end{abstract}

(c) 2016 Elsevier Ltd. All rights reserved.

\section{Introduction}

Most rigorous process design problems are carried out under a deterministic setting with fixed specifications. In reality, however, the process inputs and parameters exhibit some randomness as depicted in Fig. 1, which can have a significant effect on the safety, reliability and economic decisions. Therefore, it is important to examine the effects of these uncertainties and analyze the sensitivity of the process model with respect to these uncertainties in the design stage. Monte-Carlo (MC) and Quasi Monte-Carlo (QMC) methods are representative probabilistic approaches for the propagation of uncertainties in the model input to its output (Niederreiter et al., 1996; Liu, 2001; Kroese et al., 2011; Abubakar et al., 2015). The brute-force implementation of these models first involves the generation of an ensemble of random realizations with each parameter drawn randomly from its uncertainty distribution. Deterministic solvers are then applied to each member to obtain an ensemble of results. The ensemble of results is then post-processed to estimate

\footnotetext{
* Corresponding author. Fax: +82 538113262 .

E-mail address: mynlee@yu.ac.kr (M. Lee).
}

the relevant statistical properties, such as the mean, standard deviation and quantile. Despite this, estimations of the mean converge with the inverse square root of the number of runs, making MCand QMC-based approaches computationally expensive and even infeasible for complex chemical process problems.

Recently, uncertainty analysis using a generalized polynomial chaos (gPC) expansion was studied in various applications including modeling, control, robust optimal design, and fault detection problems. Nagy and Braatz (2007) considered the gPC approach for uncertainty quantification and robust design of batch crystalized process. In their work, it was shown that the gPC approach to be more computationally efficient than the MC/QMC methods for a system with a moderate number of random inputs. Duong and Lee (2012, 2014) applied the gPC method to the PID controller design for fractional order and integer order systems. Du et al. (2015) considered the fault detection problem by combining maximum likelihood with the gPC framework. The gPC method originated from Wiener chaos (Wiener, 1938). This method is a spectral representation of a random process by the orthonormal polynomials of a random variable. Exponential convergence is expected for the gPC expansion of infinitely smooth functions (i.e., analytic and infinitely differentiable). Ghanem and Spanos (1991) reported that the gPC 


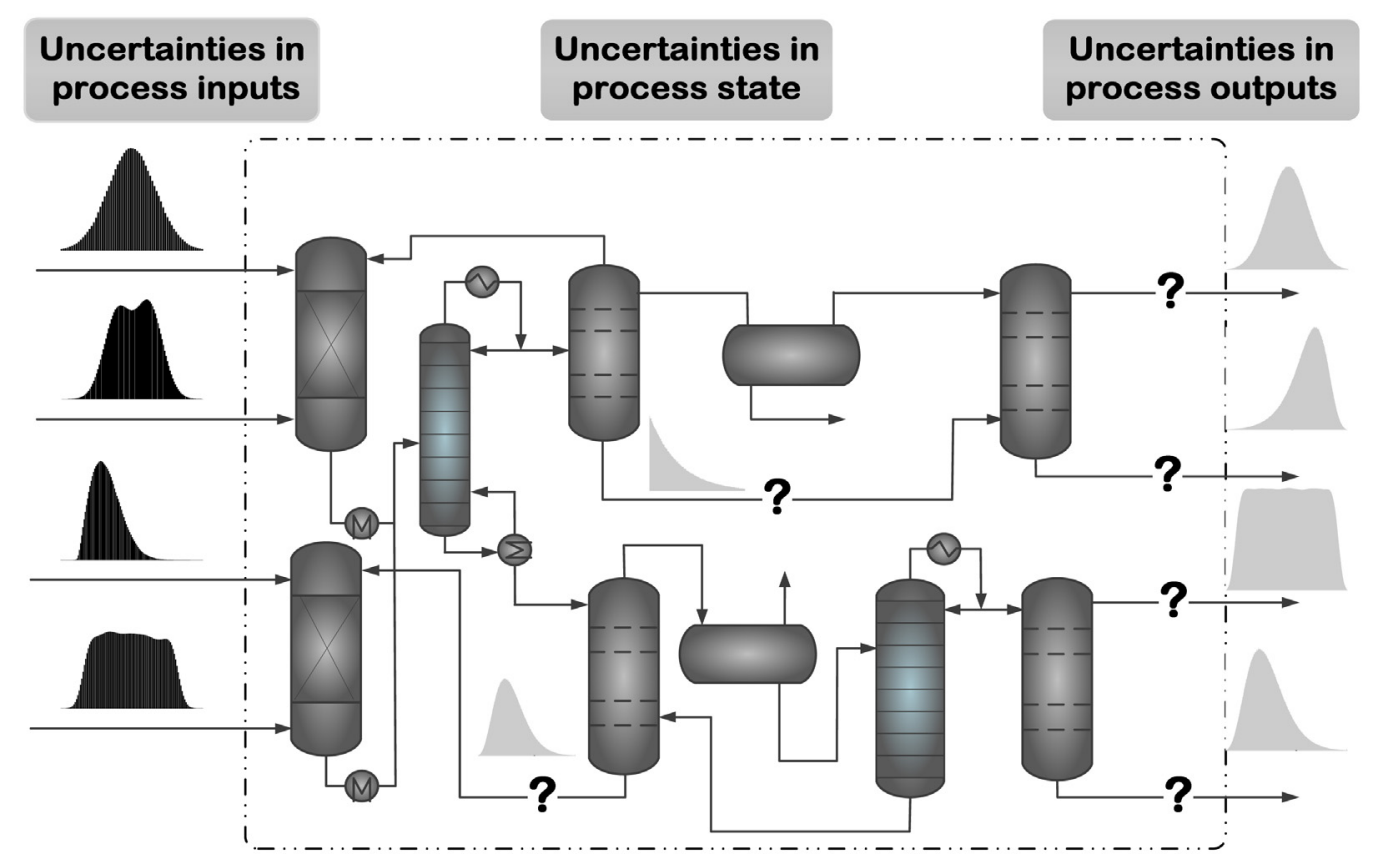

Fig 1. Uncertainty propagation and quantification in chemical processes.

is an effective computational tool for engineering purposes. Xiu and Karniadakis (2002) further generalized PC for use with nonstandard distributions.

This work demonstrates and validates the applicability of polynomial chaos theory for uncertainty quantification and sensitivity analysis for complex chemical processes such as natural gas and syngas production. The proposed gPC based method can reduce significantly the computational cost (simulation time) for uncertainty quantification over traditional approaches, such as the MC/QMC methods. Moreover, Sobol's sensitivity indices (Sobol, 2001) can also be directly obtained from the gPC surrogate analytical model (Crestaux et al., 2009; Sandoval et al., 2012), which can in turn be used to detect the influential inputs in the propagation of process uncertainty.

\section{Uncertainty quantification using polynomial chaos theory}

Consider a steady-state process that is described with a set of following nonlinear equations:

$\mathbf{F}(\mathbf{y}, \xi)=\mathbf{0}$

where $\xi=\left(\xi_{1}, \xi_{2}, \ldots \xi_{N}\right)$ is a process input variable vector expressed by a random vector of mutually independent random components with probability density functions of $\rho_{i}\left(\xi_{i}\right): \Gamma_{i} \rightarrow R^{+}$; and $\boldsymbol{y}$ denotes a process state and output variable vector.

The joint probability density of the random vector, $\xi$, is $\rho=$ $\prod_{i=1}^{N} \rho_{i}$, and the support of $\boldsymbol{\xi}$ is $\boldsymbol{\Gamma} \equiv \prod_{i=1}^{N} \Gamma_{i} \in R^{N}$. The uncertainties in the process inputs $\xi$ are then propagated through the entire process, as shown in Fig. 1. The set of one-dimensional orthonormal polynomials, $\left\{\phi_{i}\left(\xi_{i}\right)_{m=0}^{d_{i}}\right\}$, can be defined in finite dimension space, $\Gamma_{i}$, with respect to the weight, $\rho_{i}\left(\xi_{i}\right)$. Based on a one-dimensional set of polynomials, an $N$-variate orthonormal set can be constructed with $P$ total degrees in space, $\Gamma$, using the tensor product of the one- dimensional polynomials, the basis function of which satisfies the following:

$\int_{\boldsymbol{\Gamma}} \Phi_{m}(\xi) \Phi_{n}(\xi) \rho(\xi) \mathrm{d} \xi=\left\{\begin{array}{l}1, m=n \\ 0, m \neq n\end{array}\right.$

Consider a response function $f(y(\xi))$ for a process state variable, $y$, with the statistics (e.g., mean, variance) of interest, the $\mathrm{N}$-variate $P^{\text {th }}$ order approximation of the response function can be constructed as follows:

$f_{N}{ }^{P}(y(\xi))=\sum_{i=1}^{M} \hat{f}_{i} \Phi_{i}(\xi)$

$M+1=\left(\begin{array}{c}N+P \\ N\end{array}\right)=\frac{(N+P) !}{N ! P !}$

where $P$ is the order of polynomial chaos, and $\hat{f}_{m}$ is the coefficient of gPC expansion that satisfies Eq. (2) as follows:

$\hat{f}_{i}=\boldsymbol{E}\left[\Phi_{i} f(y)\right]=\int_{\Gamma} f(y) \Phi_{i}(\boldsymbol{\xi}) \rho(\boldsymbol{\xi}) \mathrm{d} \boldsymbol{\xi}$

where $\boldsymbol{E}[\cdot]$ denotes the expectation operator.

The coefficients of the gPC expansion from Eq. (4) are normally obtained numerically using the following procedure (Xiu, 2010):

- Choose a $\mathrm{N}$-dimensional integration rule (cubature nodes and weights)

$$
\begin{gathered}
\ell^{Q}[g]=\left(Q_{q_{1}}{ }^{(1)} \otimes \ldots \otimes Q_{q_{N}}{ }^{(N)}\right)[g]=\sum_{j_{1}=1}^{q_{1}} \ldots \sum_{j_{N}=1}^{q_{N}} g\left(\xi_{j}^{\left(j_{1}\right)}, \ldots, \xi_{j}^{\left(j_{N}\right)}\right) \\
\left(w_{1}{ }^{\left(j_{1}\right)} \ldots w_{1}{ }^{\left(j_{N}\right)}\right) \cong \int_{\Gamma} g(\xi) \rho(\xi) \mathrm{d} \xi
\end{gathered}
$$

where $\otimes$ denotes the tensor product, and $\ell^{\mathrm{Q}}[\cdot]$ denotes the multivariate cubature approximation.

- Approximate the gPC coefficients in Eq. (4) using the numerical integration rule in Eq. (5). 
$\tilde{\hat{f}}_{i}=\ell^{Q}[f(y, \xi) \Phi(\xi) \rho]=\sum_{m=1}^{Q} f\left(\xi^{(m)}\right) \Phi_{j}\left(\xi^{(m)}\right) w^{(m)}$ for $j=1, \ldots, M$

where $\tilde{\hat{f}}$ represents the numerical approximation of $\hat{f}$ and $f(\xi) \Phi_{j}(\xi)$ plays the role of $g(\xi)$ in Eq. (5); $Q$ is the number of nodes in cubature approximation. The number of cubature nodes (simulations) rises exponentially and hence the tensor cubature should be used only for small number of parameters. However, for moderate number of parameters (5-10 parameters), a sparse grid quadrature can be used for calculating the gPC in Eq. (6) (Crestaux et al., 2009; Xiu, 2010).

- Construct an $N$-variate $P^{\text {th }}$ order gPC approximation of the response function in the form:

$\tilde{f}_{N}^{P}=\sum_{j=1}^{M} \tilde{\hat{f}}_{j} \Phi_{j}(\xi)$

Once all the gPC coefficients and the surrogate model in Eq. (7) have been obtained, a post-processing procedure is then carried out to obtain the statistical properties of the response function $f(y(\xi)$ ).

The first expansion coefficient is the mean value as follows:

$\boldsymbol{E}\left[\tilde{f}_{N}^{P}\right]=\mu_{f}=\int_{\Gamma} \tilde{f}_{N}^{P} \rho(\boldsymbol{\xi}) \mathrm{d} \boldsymbol{\xi}=\int_{\Gamma}\left[\sum_{j=1}^{M} \tilde{\hat{f}}_{j}(\boldsymbol{\xi}) \Phi_{j}(\boldsymbol{\xi})\right] \rho(\boldsymbol{\xi}) \mathrm{d} \boldsymbol{\xi}=\tilde{\hat{f}}_{1}$

The variance of the response function $f(y(\xi))$ can be evaluated as follows:

$$
\begin{aligned}
& D_{f}=\sigma^{2}=\mathrm{E}\left[\left(f-\mu_{f}\right)^{2}\right] \\
& =\int_{\Gamma}\left(\sum_{j=1}^{M} \tilde{\hat{f}}_{j}(\xi) \Phi_{j}(\xi)-\tilde{\hat{f}}_{j}\right)\left(\sum_{j=1}^{M} \tilde{\hat{f}}_{j}(\xi) \Phi_{j}(\xi)-\tilde{\hat{f}}_{1}\right) \rho(\xi) \mathrm{d} \xi=\sum_{j=2}^{M} \tilde{\hat{f}}_{j}^{2}
\end{aligned}
$$

Eqs. (8) and (9) employ the property that the polynomial set begins with $\Phi_{1}(\xi)=1$. The weight function of the polynomial is the probability density function.

The distribution function of the response function is obtained by sampling the surrogate model in Eq. (7).

If a response function $f(y)$ is chosen for as state variable, $y$, the mean and variance of the system's states are given approximately by Eqs. (8) and (9), respectively. Duong and Lee (2012) and the references therein present different methods for constructing a set of orthonormal polynomials with respect to a given distribution.

Remark. In the MC/QMC methods, it is necessary to simulate the process numerous times at the sampling points to obtain the required probability. On the other hand, the gPC requires solving Eq. (1) only at the cubature nodes for obtaining the analytical representation in Eq. (7). The analytical model by Eq. (7) can be sampled easily with little simulation effort. The statistical moments are available directly from the surrogate model, as expressed in Eqs. (8) and (9).

\section{Variance base sensitivity analysis}

\subsection{Brief theory of variance base sensitivity analysis}

In addition to the uncertainty quantification, global sensitivity analysis is an essential step to identify the parameters that are relatively more important than the others over the entire parameter space of the models in the science and engineering fields. One popular approach for sensitivity analysis is variance based; its theory is given briefly in this section.

Consider the system described in Fig. 1 and Eq. (1). The mean and variance of the response function $f(y(\xi))$ can be expressed as

$\mu_{f_{y}}=\int_{\Gamma_{1}} \ldots \int_{\Gamma_{N}} f\left(y\left(\xi_{1}, \ldots, \xi_{N}\right)\right) \prod_{i=1}^{N} \rho_{i}\left(\xi_{i}\right) \mathrm{d} \xi_{i}$

$D_{f_{y}}=\int_{\Gamma_{1}} \ldots \int_{\Gamma_{N}}\left[f\left(y\left(\xi_{1}, \ldots, \xi_{N}\right)\right)-\mu_{f_{y}}\right]^{2} \prod_{i=1}^{N} \rho_{i}\left(\xi_{i}\right) \mathrm{d} \xi_{i}$.

The response function of the system output can be decomposed into sum of terms with increasing dimensions (Satelli et al., 2004):

$f(y(\xi))=f_{0}+\sum_{i=1}^{N} f_{i}\left(\xi_{i}\right)+\sum_{i=1}^{N-1} \sum_{j>i}^{N} f_{i j}\left(\xi_{i}, \xi_{j}\right)+\ldots+f_{i_{1} \ldots i_{N}}\left(\xi_{i}, \ldots, \xi_{N}\right)$,

where $f_{0}=\mu f_{y}$.

The terms in Eq. (12) are functions of the factors in its index and can be expressed as

$f_{i}\left(\xi_{i}\right)=\mathbb{E}\left[f(y(\xi)) \mid \xi_{i}\right]-\mu_{f}$

$f_{i j}\left(\xi_{i}, \xi_{j}\right)=\mathbb{E}\left[f(y(\xi)) \mid \xi_{i}, \xi_{j}\right]-f_{i}-f_{j}-\mu_{f}$,

where $\mathbb{E}\left[f(\mathrm{y}(\xi)) \mid \xi_{i}\right]$ (resp. $\left.\mathbb{E}\left[f(\mathrm{y}(\xi)) \mid \xi_{i} \xi_{j}\right]\right)$ is the conditional expectation of $f(y(\xi))$ when $\xi_{i}$ is set (resp. $\xi_{i}$ and $\xi_{j}$ are set). The decomposition in Eqs. (12) and (13) is unique provided that the random factors are independent.

Therefore, the variance of the output function can be decomposed as follows:

$D_{f_{y}}=\sum_{i=1}^{N} D_{i}+\sum_{i=1}^{N-1} \sum_{j>i}^{N} D_{i j}+\ldots+D_{1,2, \ldots, N}$

where

$D_{i}=\operatorname{var}\left(\mathbb{E}\left[f(y(\xi)) \mid \xi_{i}\right]\right)$

$D_{i j}=\operatorname{var}\left(\mathbb{E}\left[f(y(\xi)) \mid \xi_{i}, \xi_{j}\right]\right)-D_{i}-D_{j}$

$D_{1,2, \ldots, N}=D_{f_{y}}-\sum_{i=1}^{N} D_{i}-\ldots-\sum_{1 \leq i_{1}<\cdots<i_{n-1} \leq N} D_{i_{1} \ldots i_{N-1}}$

Note that in $\operatorname{var}\left(\mathbb{E}\left[f(y(\xi)) \mid \xi_{i}, \xi_{j}\right]\right)$, the inner expectation is over all factors except for $\xi_{i}, \xi_{j}$, and the outer variance is over $\xi_{i}, \xi_{j}$.

The first order sensitivity index (function) is defined as follows:

$S_{i}=\frac{D_{i}}{D_{f_{y}}}$

The first order index represents the main effect contribution of each random input factor to the variance of the output alone. $S_{i}$ lies in $[0,1]$. The sum of the first order functions equals 1 for the additive models.

Similarly, let the sensitivity functions of a higher order be defined as

$S_{i_{1}, \ldots, i_{k}}=\frac{D_{i_{1}, \ldots, i_{k}}}{D_{f_{y}}}$ 


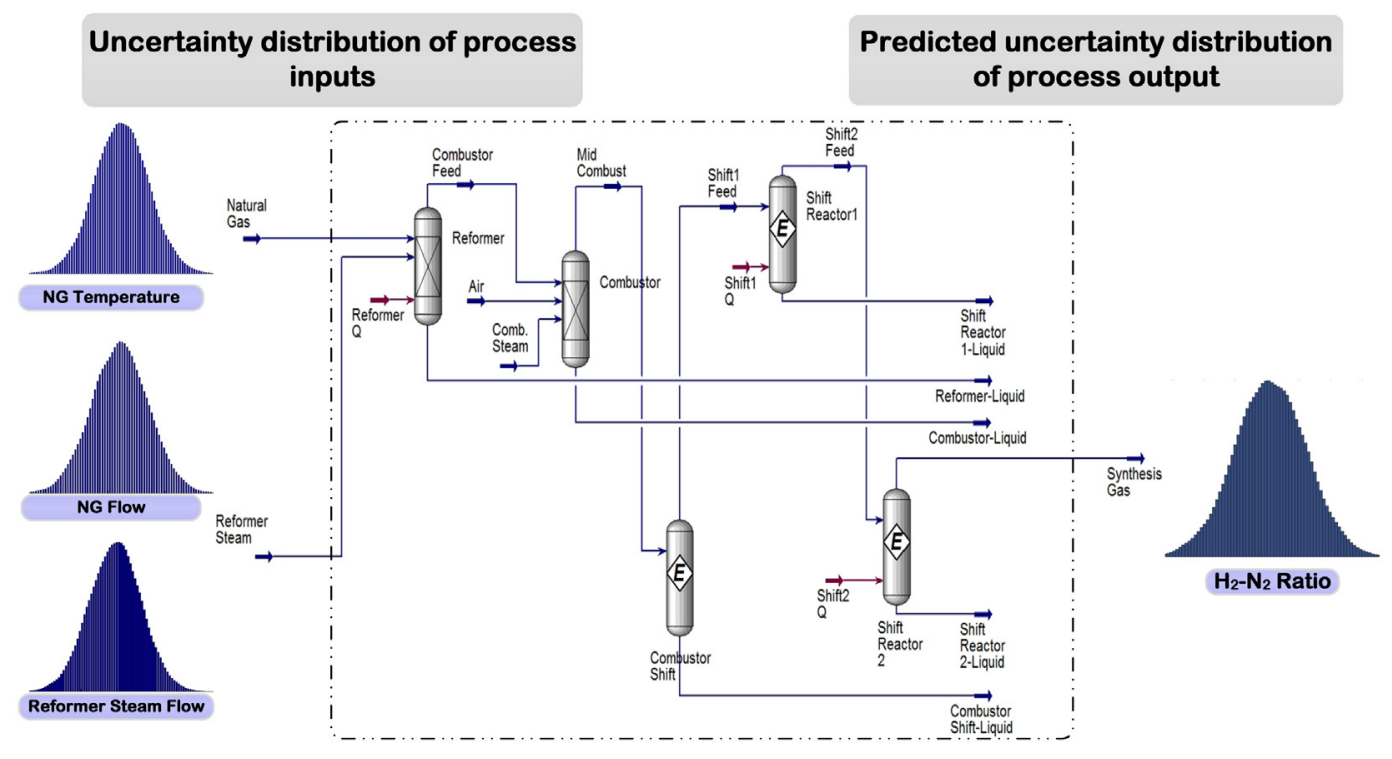

Fig. 2. Syngas production process (Example 1).

The sensitivity function of a higher order is a sensitivity measure that describes what part of the total variance is due to the uncertainties in the set of parameters, $\left\{\xi_{i_{1}}, \ldots, \xi_{i_{s}}\right\}$.

The total effect function for the factor, $\xi_{i}$, can be written as

$T_{i}=1-\frac{\operatorname{var}\left(\mathbb{E}\left(f_{y}(\boldsymbol{\xi}) \mid \boldsymbol{\xi}_{\sim i}\right)\right)}{D_{f_{y}}(t)}$.

This total effect index measures the contribution to the output variance of $\xi_{i}$, including all variances caused by its interactions, of any order, with any other input variables. The function, $T_{i}$, is the educated answer to the question, "Which factor can be fixed anywhere over its range of variability without affecting the output?" (Satelli et al., 2004, 2008).

\subsection{Variance based sensitivity analysis using $g P C$}

The gPC expansions in Eq. (7) can be reordered to separate the single and collective contribution of each parameter as follows.

Define the set of multi-indices $I_{k_{1}, \ldots, k_{s}}$ such that (Sandoval et al., 2012):

$I_{k_{1}, \ldots, k_{\mathrm{s}}}=\left\{\left(k_{1}, k_{2}, \ldots, k_{\mathrm{s}}\right): 0 \leq \gamma_{k}^{j} \leq P, \gamma_{k}^{j}=0, k \in\{1, \ldots, n\} \backslash\left\{k_{1}, \ldots, k_{\mathrm{s}}\right\}\right\}$

where $\gamma_{k}^{j}$ is the one-dimensional polynomial degree. Using this notation, the first order sensitivity function can be expressed as

$S_{i}=\frac{\sum_{j \in I_{i}} \hat{f}_{j}^{2}}{D_{f}}$.

The estimated sensitivity function of a higher order can be obtained in the same manner as follows:

$S_{i_{1}, \ldots, i_{s}}=\frac{\sum_{j \in I_{i_{1}, \ldots, i_{s}}} \hat{f}_{j}^{2}}{D_{f}}$.

Remark. Normally, for estimation of first order and total sensitivity indices the computational complexity is $(N+2) \times M$, where $N$ is the number of variables, and $M$ is the number of the MC/QMC samples (Satelli et al., 2004, 2008). Note that only M samples are needed for uncertainty quantification problem.

\section{Chemical process examples}

In this section, the proposed gPC based method was applied to uncertainty quantification and sensitivity analysis of two chemical process examples studied in (Abubakar et al., 2015), such as a syngas production process and a sales gas process, and validated using standard MC/QMC methods.

\subsection{Example 1.a: syngas production process with Gaussian uncertainties}

Fig. 2 shows a process flow diagram of a synthesis gas (or alternatively known as syngas) production process. In this process, natural gas (NG) rich in methane enters the reformer, where a large amount of methane reacts with steam and produces syngas with $\mathrm{CO}_{2}$. The effluent from the reformer is then sent to the combustor with an air stream. Steam is also fed into the combustor to maintain the reactor temperature and ensure the complete combustion of methane. The product from the combustor passes through the shift reactors, where the water gas shift reactions are carried out. The synthesis gas is finally taken as the feedstock for an ammonia production plant. To facilitate the production of ammonia, the hydrogen to nitrogen molar ratio $(\mathrm{HNr})$ in the syngas needs to be controlled precisely at 3:1, which represents the stoichiometric amounts of the reactants in the ammonia process.

In this example, the NG feed temperature, flow rate, and the reformer steam flow rate were assumed to be uncertain and normally distributed independently with a mean and standard deviation of $\left\{370^{\circ} \mathrm{C}, 100 \mathrm{~kg} \mathrm{~mol} / \mathrm{h}, 240 \mathrm{~kg} \mathrm{~mol} / \mathrm{h}\right\}$ and $\left\{55.5^{\circ} \mathrm{C}\right.$, $15 \mathrm{~kg} \mathrm{~mol} / \mathrm{h}, 36 \mathrm{~kg} \mathrm{~mol} / \mathrm{h}\}$, respectively. All other process inputs (such as air stream and combustion steam to the combustor) and process parameters (such as the vessel volume, heat duty and pressure) were assumed to be deterministic. A code to generate the cubature nodes and weights in the gPC method was developed in a Matlab ${ }^{\mathrm{TM}}$ environment and connected to HYSYS ${ }^{\mathrm{TM}}$, where the syngas process in Fig. 2 was modeled rigorously. The cubature nodes from gPC code are passed to HYSYS ${ }^{\mathrm{TM}}$, and the impact of the uncertainties on the hydrogen-nitrogen ratio ( $\mathrm{HNr}$ ) was monitored. The proposed gPC method (5th order gPC) used 125 Gauss Hermite cubature nodes. From the simulation result obtained with the cubature nodes, the surrogate gPC model was obtained for $\mathrm{HNr}$ using the theory described in Section 2. It is well known that the 
MC/QMC methods cannot offer an accurate result with a low number of samples. Since true estimates of the output for the studied process are not available, the result from the proposed method was compared with those from the MC/QMC methods with a sufficiently large number of samples. Table 1 lists the statistical properties of $\mathrm{HNr}$ obtained from the gPC method and the conventional MC/QMC methods. Fig. 3 compares the density functions of $\mathrm{HNr}$ obtained from the $\mathrm{gPC} / \mathrm{MC} / \mathrm{QMC}$ methods. The results from the proposed gPC method matched those from the traditional MC and QMC methods. Note that the number of simulations required by the proposed gPC method was significantly lower than those by the other two methods. As shown in Fig. 3, the traditional MC and/or QMC methods require a huge number of samples to get similar results. It is noted that the performance of the gPC method may degrade for high order statistical moments of non-smooth quantity of interest (output).

Another unique advantage of the proposed gPC method is that Sobol's sensitivity indices to identify the influential inputs in the propagation of process uncertainty can be obtained directly from the surrogate analytical model, which can be used to reduce the number of simulations required from 10-100 times, and the computational efforts needed for the uncertainty quantification. In this example, the sensitivity indices for the process variable $\mathrm{HNr}$ with respect to three uncertain inputs (the NG feed temperature and flow rate, the reformer steam flow rate) can be obtained from the surrogate gPC model. Table 2 lists the sensitivity indices obtained from the surrogate gPC model. The only input that matters is the second one, i.e., the NG feed flow rate. The natural gas temperature and the reformer steam flow are non-influential and can be excluded from the analysis of uncertainty propagation. Fig. 4 compares density distributions predicted by the gPC and QMC methods with one random parameter (i.e., the NG feed flow rate) and that by the QMC method (with 10,000 simulations from Halton sequence) with all three random parameters. Note that in the case of one random input, only five simulations were used for the proposed gPC method, whereas 10,000 simulations for the QMC method. The density functions from both methods with one random variable showed a close match with that using all three random variables. The number of samples for the MC/QMC methods was chosen according to Chernoff bound (Tempo et al., 2013) for accurate estimation of the probability.

\subsection{Example 1.b: syngas production process with uniform uncertainties}

In this example, all the three process inputs of the syngas production process in Fig. 2 were assumed to have independent uncertainties distributed uniformly in intervals of $\left\{370 \pm 50^{\circ} \mathrm{C}\right.$, $100 \pm 20 \mathrm{~kg} \mathrm{~mol} / \mathrm{h}, 240 \pm 54 \mathrm{~kg} \mathrm{~mol} / \mathrm{h}\}$. The Gauss Legendre cubature nodes were used to obtain the surrogate gPC model, as described in Section 2. The result from the proposed gPC method was then compared with the traditional MC and QMC methods. Tables 1 and 2 list the statistical properties of the $\mathrm{HNr}$ and the sensitivity indices, respectively.

Fig. 5 compares the density functions predicted using the proposed gPC method (7th order gPC with 343 simulations), the QMC method with 16,000 simulations and the MC method with 1000 simulations. The MC method with a low number of samples cannot provide an accurate result, even though the number of samples was much larger than that used in the gPC method. The result by the gPC method showed a close match with that using the QMC method with a large number of samples. Sobol's indices in Table 2 also showed that the important random parameter influencing the uncertainty propagation was only a NG feed flow rate and two other random inputs can be excluded from the analysis. Fig. 6 compares the density function of $\mathrm{HNr}$ with three random inputs and those with one random input (i.e., the NG feed flow), whereas the other

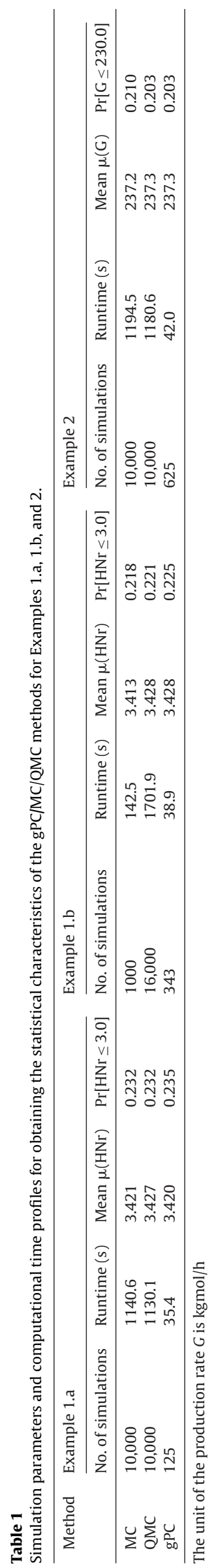


Table 2

Sobol's sensitivity indices from the surrogated gPC model for Examples 1.a, 1.b, and 2.

\begin{tabular}{|c|c|c|c|c|c|c|c|c|}
\hline \multicolumn{9}{|c|}{ Sobol's sensitivity indices $\left(S_{i j \ldots n}\right)$} \\
\hline Example 1.a & $\begin{array}{l}S_{1} \\
2.272 \mathrm{E}-05\end{array}$ & $\begin{array}{l}S_{2} \\
0.9806\end{array}$ & $\begin{array}{l}S_{3} \\
0.0137\end{array}$ & $\begin{array}{l}S_{12} \\
1.646 \mathrm{E}-05\end{array}$ & $\begin{array}{l}S_{13} \\
0.0020\end{array}$ & $\begin{array}{l}S_{23} \\
0.0022\end{array}$ & $\begin{array}{l}S_{123} \\
0.0014\end{array}$ & \\
\hline Example 1.b & $\begin{array}{l}S_{1} \\
4.940 \mathrm{E}-05\end{array}$ & $\begin{array}{l}S_{2} \\
0.9479\end{array}$ & $\begin{array}{l}S_{3} \\
0.0062\end{array}$ & $\begin{array}{l}S_{12} \\
5.220 \mathrm{E}-04\end{array}$ & $\begin{array}{l}S_{13} \\
8.840 \mathrm{E}-04\end{array}$ & $\begin{array}{l}S_{23} \\
0.0371\end{array}$ & $\begin{array}{l}S_{123} \\
0.0074\end{array}$ & \\
\hline Example 2 & $\begin{array}{l}S_{1} \\
0.9036 \\
S_{24} \\
3.2377 \text { E-09 }\end{array}$ & $\begin{array}{l}S_{2} \\
0.0795 \\
S_{34} \\
1.8833 \mathrm{E}-09\end{array}$ & $\begin{array}{l}S_{3} \\
0.0167 \\
S_{123} \\
5.4235 \mathrm{E}-08\end{array}$ & $\begin{array}{l}S_{4} \\
9.7715 \mathrm{E}-06 \\
S_{124} \\
3.9457 \mathrm{E}-14\end{array}$ & $\begin{array}{l}S_{12} \\
6.7285 \mathrm{E}-05 \\
S_{134} \\
7.5100 \mathrm{E}-14\end{array}$ & $\begin{array}{l}S_{13} \\
2.5839 \mathrm{E}-06 \\
S_{234} \\
5.8284 \mathrm{E}-12\end{array}$ & $\begin{array}{l}S_{14} \\
1.8018 \mathrm{E}-12 \\
S_{1234} \\
4.6192 \mathrm{E}-14\end{array}$ & $\begin{array}{l}S_{23} \\
6.4715 \mathrm{E}-05\end{array}$ \\
\hline
\end{tabular}

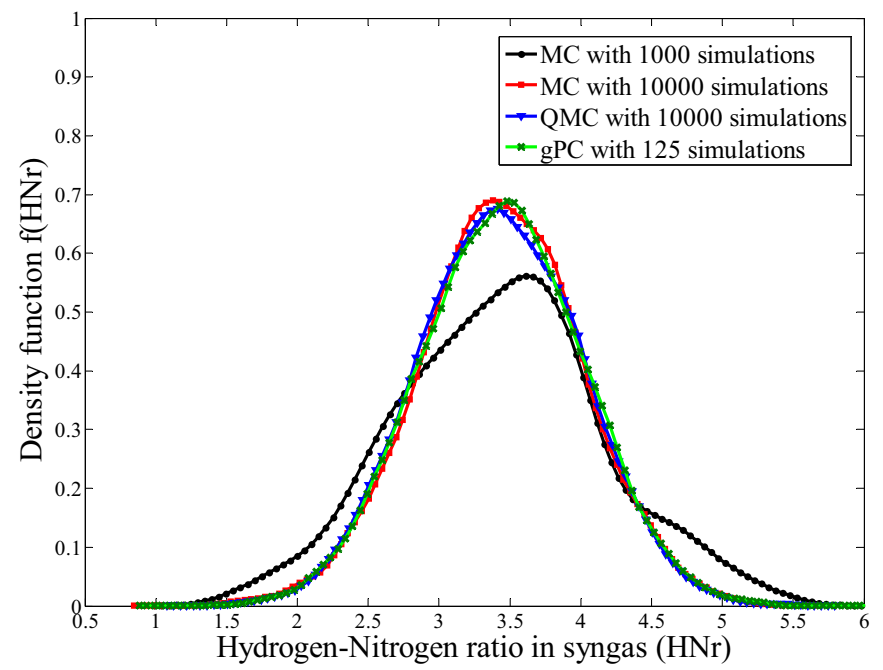

Fig. 3. Density distributions of the hydrogen-nitrogen ratio in syngas with 3 Gaussian random inputs (Example 1.a).

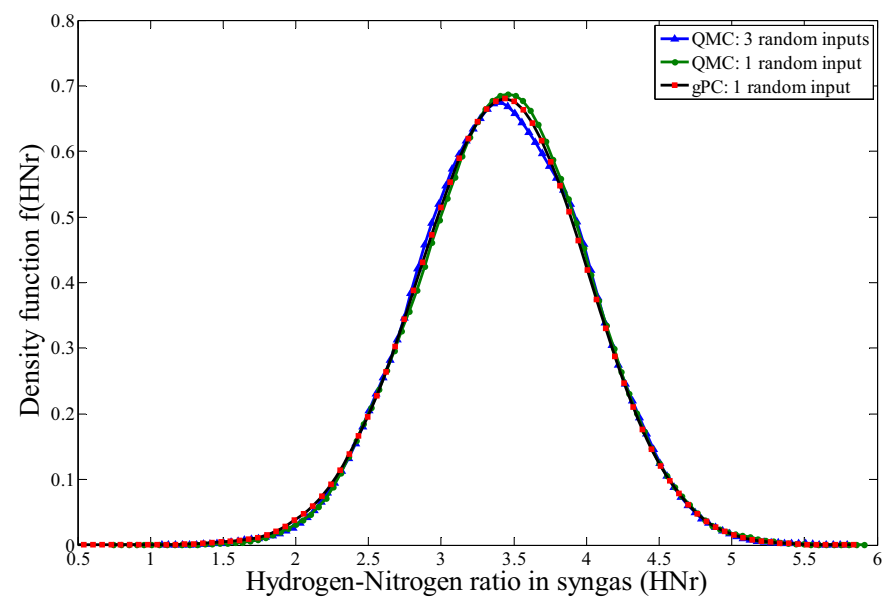

Fig. 4. Density distributions of the hydrogen-nitrogen ratio in syngas with 3 and 1 Gaussian random inputs (Example 1.a).

two inputs are assumed to be constant at their mean values. As shown in the figure, the results using the important random input only were identical to those using all three random inputs. The proposed gPC method with one random input required only seven simulations, whereas the QMC method used 16,000 simulations to obtain an accurate result. Note that for calculating sensitivity indices using the QMC method, one may need $16,000 \times 5$ simulations. Hence due to the large computational burden for calculating sensitivity indices by the QMC method, we will not do the QMC approach for validation of the gPC method. However, Fig. 6 shows that the gPC approach correctly detects non- influential input.

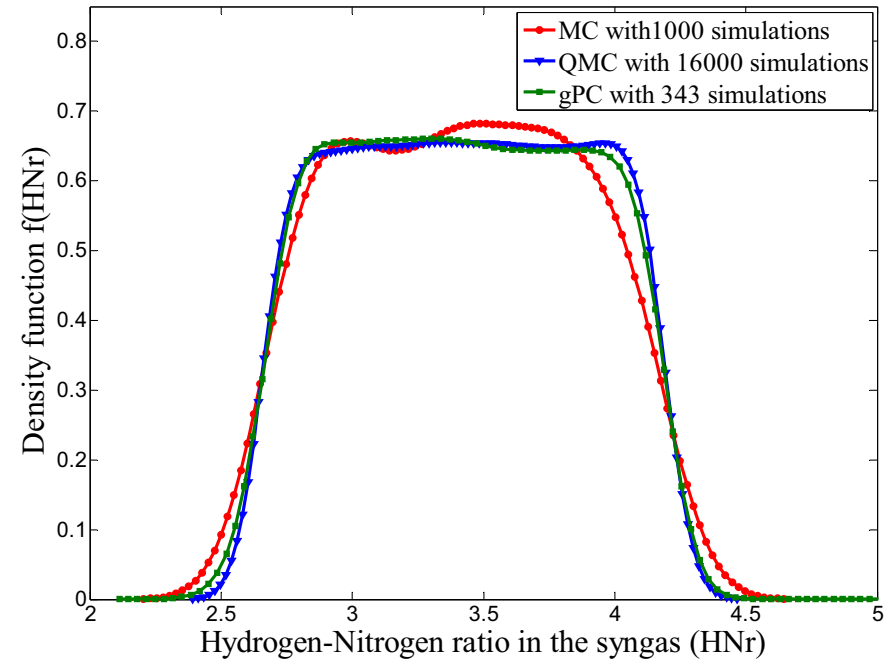

Fig. 5. Density distributions of the hydrogen-nitrogen ratio in syngas with 3 uniform random inputs (Example 1.b).

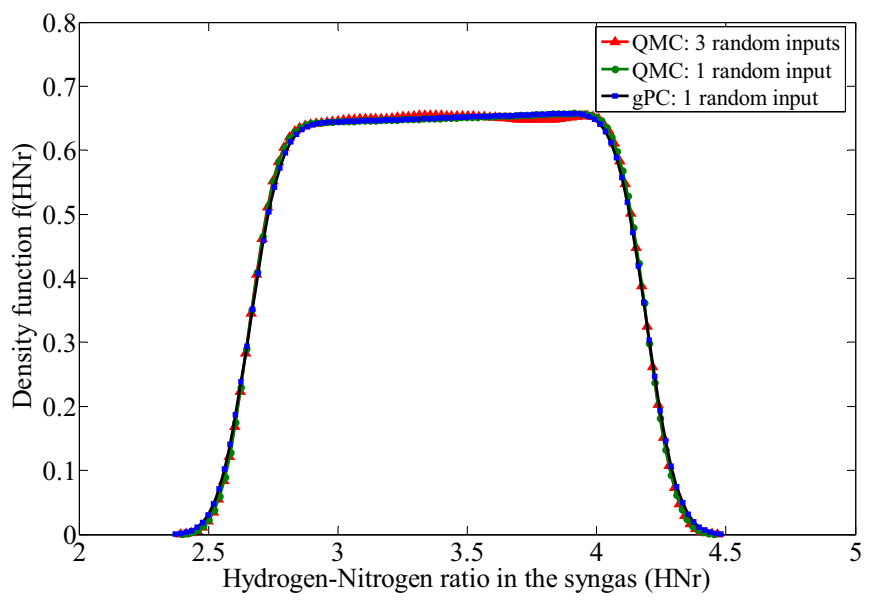

Fig. 6. Density distributions of the hydrogen-nitrogen ratio in syngas with 3 and 1 uniform random inputs (Example 1.b).

\subsection{Example 2: sales gas process with Gaussian uncertainties}

Fig. 7 shows a process flow diagram of a sales gas process. In this process, the crude natural gas is refined to a lean dry sales gas to meet the hydrocarbon dew point requirements, heat duty specifications, and the production rate, $\mathrm{G}(\mathrm{kgmol} / \mathrm{h})$. The different heavy components removed from the sales gas are then fed to a depropanizer column, where propane with the remaining small amount of methane and ethane are obtained from the top, while butane with the remaining heavy components are extracted from the bottom. In this example, the crude natural gas feed flow 


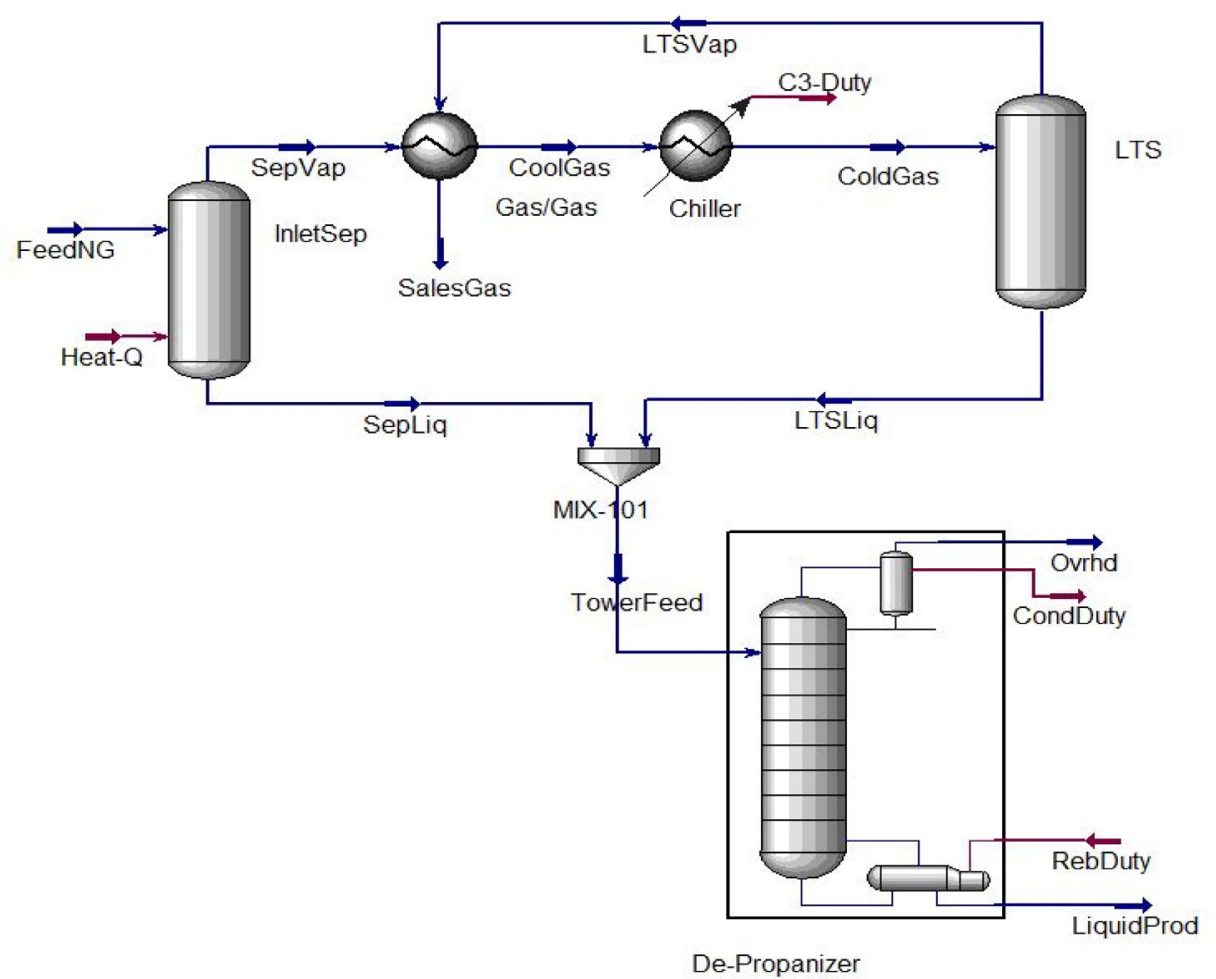

Fig. 7. Sales natural gas process (Example 2).

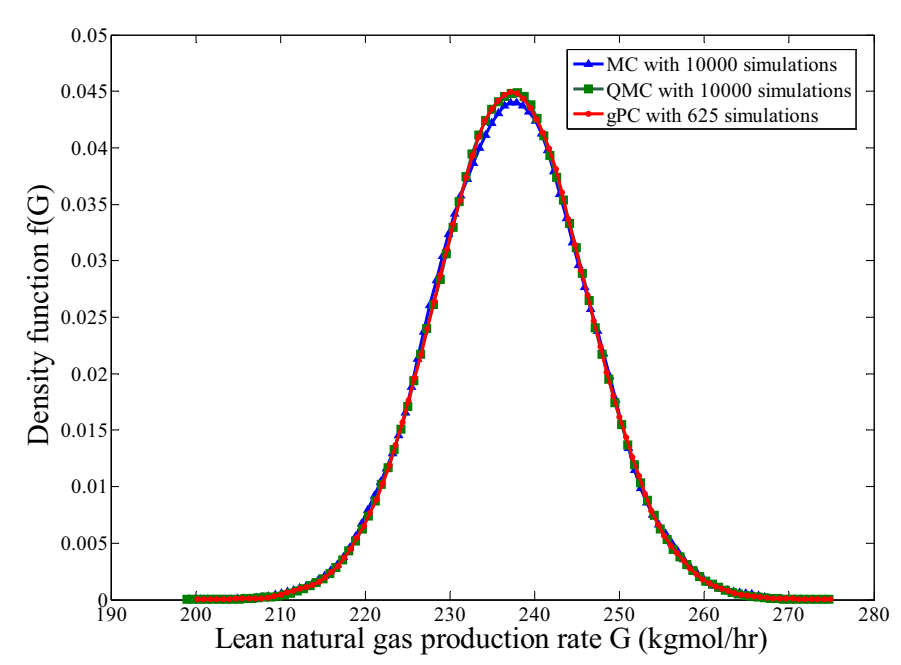

Fig. 8. Density distributions of lean gas production rate with 4 Gaussian random inputs (Example 2).

rate, pressure, temperature, and the chiller duty were assumed to be varied independently with a mean and standard deviation of $\left\{300 \mathrm{~kg} \mathrm{~mol} / \mathrm{h}, 4150 \mathrm{kPa}, 15^{\circ} \mathrm{C}, 2 \times 10^{5} \mathrm{~kJ} / \mathrm{h}\right\}$ and $\{10 \mathrm{~kg} \mathrm{~mol} / \mathrm{h}$, $\left.150 \mathrm{kPa}, 3^{\circ} \mathrm{C}, 500 \mathrm{~kJ} / \mathrm{h}\right\}$, respectively. Fig. 8 shows the density functions for lean dry gas production predicted from the $\mathrm{gPC} / \mathrm{MC} / \mathrm{QMC}$ methods. The result from the gPC method (5th order gPC with 625 simulations) closely matched the result from the MC/QMC methods with 10,000 simulations. Table 1 lists the statistical properties of lean gas production and simulation parameters from the proposed gPC and MC/QMC methods. Table 2 lists the sensitivity indices obtained from the surrogate gPC model. The sensitivity indices indicate that the crude NG feed flow rate and pressure are influential for uncertainty propagation while the crude NG feed temperature and the chiller duty are non-influential. Fig. 9 shows the density func-

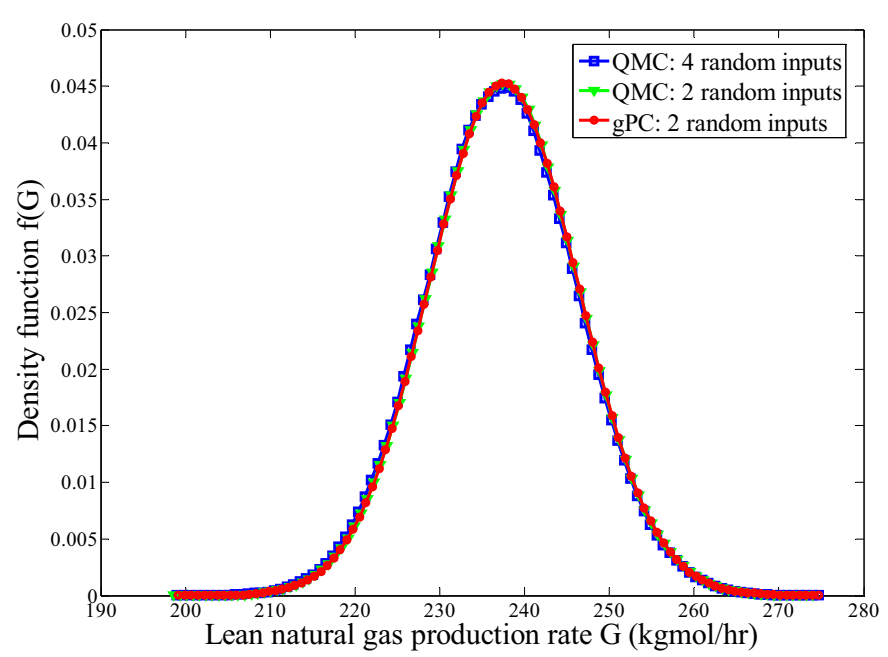

Fig. 9. Density distributions of lean gas production rate with 4 and 2 Gaussian random inputs (Example 2 ).

tions of lean gas production with two influential random inputs by the gPC method (with 25 simulations) and by the QMC method (with 10,000 simulations), and compares with that using all four random parameters by the QMC method (with 10,000 simulations). The sensitivity indices from the gPC method correctly identify the influential inputs.

\section{Conclusions}

A stochastic spectral approach based method was proposed to model the uncertainty propagation and sensitivity in complex chemical processes. The gPC method was applied to determine the solution to these problems. An interface between Matlab ${ }^{\mathrm{TM}}$ and HYSYS $^{\mathrm{TM}}$ was used to provide a rigorous result in all simulations. 
The results showed precise agreement with those of the conventional approaches such as the MC/QMC methods, which might be beyond the computational capability for large scale complex chemical process problems. The gPC approach has advantages over the popular MC/QMC approaches, mainly in terms of the computational cost: the required number of samples for the uncertainty prediction can be reduced significantly by the gPC method. Moreover, Sobol's sensitivity indices to identify the influential random inputs for uncertainty propagation can be obtained directly from the surrogated gPC model, which in turn reduces the required samples remarkably and allows a focus on the important inputs for the robust design of complex processes under a range of uncertainties.

\section{Acknowledgments}

This study was supported by the Basic Science Research Program through the National Research Foundation of Korea (NRF) funded by the Ministry of Education(2015R1D1A3A01015621), and also by the Priority Research Centers Program through the National Research Foundation of Korea (NRF) funded by the Ministry of Education (2014R1A6A1031189).

\section{References}

Abubakar, U., Srirnivas Sriramula, S., Renton, C., 2015. Reliability analysis of complex chemical engineering processes. Comput. Chem. Eng. 74, 1-14.

Crestaux, T., Le Maitre, O., Martinez, J.M., 2009. Polynomial chaos for sensitivity analysis. Reliab. Eng. Syst. Saf. 94 (7), 1161-1172.

Du, Y., Duever, T.A., Budman, H., 2015. Fault detection and diagnosis with parametric uncertainty using generalized polynomial chaos. Comput. Chem. Eng. 76, 63-75.
Duong, P.L.T., Lee, M., 2012. Robust PID controller design for process with stochastic parametric uncertainties. J. Process Control 22, 1559-1566.

Duong, P.L.T., Lee, M., 2014. Probabilistic analysis and control of systems with uncertain parameters over non-hypercube domain. J. Process Control 24 358-367.

Ghanem, R.G., Spanos, P.D., 1991. Stochastic Finite Elements: A Spectral Approach. Dover Publications, New York.

Kroese, D.P., Taimre, T., Botev, Z.I., 2011. Handbook of Monte Carlo Methods. John Wiley and Sons, Hoboken, New Jersey.

Liu, J.S., 2001. Monte Carlo Strategies in Scientific Computing. Springer-Verlag, New York.

Nagy, Z.K., Braatz, 2007. Distributional uncertainty analysis using power series and polynomial chaos expansions. J. Process Control 17, 229-240, Special Issue on Advanced Control of Chemical Processes.

Niederreiter, H., Hellekalek, P., Larcher, G., Zinterhof, P., 1996. Monte Carlo and Quasi-Monte Carlo Methods. Springer-Verlag, Berlin.

Sandoval, E., Collin, F.A., Basset, M., 2012. Sensitivity study of dynamic system using polynomial chaos. Reliab. Eng. Sys. Saf. 104 (8), 15-26.

Satelli, A., Tarantola, S., Campolongo, F., Ratto, M., 2004. Sensitivity Analysis in Practice: A Guide to Assessing Scientifics Models. John Wiley \& Sons, Chichester.

Satelli, A., Ratto, M., Andres, T., Campolongo, F., Cariboni, J., Gatelli, D., Saisana, M., Tarantola, S., 2008. Global Sensitivity Analysis: The Primer. John Wiley \& Sons, Chichester.

Sobol, I.M., 2001. Global sensitivity indices for nonlinear mathematical models and their Monte Carlo estimates. Math. Comput. Simul. 55, 271-280.

Tempo, R., Calafiore, G., Dabbene, F., 2013. Randomized Algorithm for Analysis and Control of Uncertain Systems: With Applications, 2nd edition. Springer-Verlag, London.

Xiu, D., Karniadakis, G.E., 2002. The Wiener-Askey polynomial chaos for stochastic differential equations. SIAM J. Sci. Comput. 24, 619-644.

Xiu, D., 2010. Numerical Method for Stochastic Computations: A Spectral Method Approach. Princeton University Press.

Wiener, N., 1938. The homogeneous chaos. Am. J. Math 60, 897-936 